\section{Diskrete Einweghosen}

Seit Oktober 2010 sind die neuen TENA Pants mit Body-Close-FitTM auf dem Markt. Dank einem verbesserten Gewebe mit elastischen Fäden im hinteren Bereich sind die Einweghosen deutlich formstabiler und diskreter und sitzen so noch passgenauer am Körper. Die neuen TENA Pants eignen sich für Frauen und Männer jeden Alters. Mit den vier Saugstärken Discreet, Plus, Super und Maxi, die jeweils in verschiedenen Größen angeboten werden, bietet TENA das breiteste Sortiment im Markt - und somit für jeden Grad der Blasenschwäche das passende Produkt.

Nach Informationen von

SCA Hygiene, Mannheim

\section{Mehr als eine Kongress-Suchmaschine}

Die interaktive Fach-Community zur medizinischen Fort- und Weiterbildung www.my-medical-education. com ist jetzt mit neuen Funktionen online. Bekannt für die Suche medizinischer Fort- und Weiterbildungsveranstaltungen, stellt sich das Portal ab sofort als Fach-Community für alle an medizinisch-wissenschaftlicher Fortbildung interessierten Berufsgruppen vor. Das Credo des neuen, zum Social Network weiterentwickelten Portals: Finden. Bewerten. Kommunizieren. Zusätzlich zur reinen Veranstaltungssuchfunktion können Mitglieder jetzt nicht nur ihr eigenes Profil erstellen und Kontakte knüpfen, sondern aktiv Veranstaltungen sowie Referenten bewerten und kommentieren.

Mit 11.000 registrierten Ärzten und rund 90.000 erfassten nationalen und internationalen medizinischen Fort- und Weiterbildungsveranstaltungen ist my-medical-education. com das größte medizinische Portal seiner Art weltweit - und damit auch für Kongress-Veranstalter und Anbieter fachlicher Fortbildungen eine interessante Plattform, um ihre Angebote bekannt zu machen.

Nach Informationen von

Wefra, Neu-Isenburg

\title{
OAB im Alter - unbedenklich ist nur ein Anticholinergikum
}

— Benötigen ältere Patienten aufgrund einer überaktiven Blase (OAB) eine anticholinerge Therapie, dann ist hinsichtlich der Nebenwirkungen nur eine Option unbedenklich: das quartäre Amin Trospiumchlorid. Auch in einer neuen Retardformulierung erwies es sich bei älteren OAB-Patienten als wirksam, frei von Interaktionen und gut verträglich.

Wirksamkeit und Sicherheit der neuen Formulierung (urivesc ${ }^{\circledR}, 60 \mathrm{mg}$ einmal täglich) wurden in zwei randomisierten, placebokontrollierten Phase-III-Studien an insgesamt 1.165 OAB-Patienten in den USA geprüft. Das Anticholinergikum zeigte laut Dr. med. Christian Gratzke, München - die bis dahin niedrigste Inzidenz unerwünschter Ereignisse oraler antimuskarinischer Wirkstoffe in klinischen Studien und bewies in einer aktuellen Subgruppenanalyse seine Eignung vor allem für ältere, häufig von OAB betroffenen Menschen
[Sand PK et al. BJU Int 2010; 6. Aug, Epub ahead of print].

Bei 143 über 75-jährigen Patienten wurden unter der Retardformulierung im Vergleich zur Kontrollgruppe die Häufigkeit der Harnentleerungen signifikant gesenkt und das Gesamturteil der OAB-Patienten sowie ihre Lebensqualität verbesserten sich deutlich. Dieser Therapieerfolg wurde bei Patienten, die in einer Open-Lable-Studie weiterbehandelt wurden, bis zum Ende des Behandlungszeitraums nach einem Jahr beobachtet. Einen Vorteil für die Compliance sieht Gratzke in der täglichen Einmaldosis der neuen Formulierung.

Symposium „OAB Therapie - Bewährte Substanz im neuen Gewand: Trospiumchlorid als Einmalgabe“, im Rahmen des 22. Kongresses der Deutschen Kontinenz Gesellschaft e. V., Mainz, 12.11.2010

Veranstalter: Rottapharm|Madaus, Köln

\section{Jeder fünfte Mann hat niedrigen Testosteronspiegel}

_ Wie viele Männer im Alter über 40 Jahre haben einen Hypogonadismus? Die Testovital-Mess-Bar, eine mobile Messstation von Bayer Vital tourte unter dem Motto „Monat der Männergesundheit“ im September durch Frankfurt und ging dieser Frage nach. Das überraschende Ergebnis: Bei insgesamt 961 Testosteron-Checks war jeder fünfte Wert im Bereich unter $8 \mathrm{nmol} / \mathrm{l}$ und fast 30\% im Grenzbereich zwischen 8 und $12 \mathrm{nmol} / \mathrm{l}$. 810 Probanden konnten der für einen Alters-Hypogonadismus typischen Gruppe der über 40-Jährigen zugeordnet werden. Bei ihnen lagen 20\% unter $8 \mathrm{nmol} / /$ und $32 \%$ zwischen 8 und 12 nmol/l. Bemerkenswert bei den Frankfurter Untersuchungen war, dass auch von den unter 40-Jährigen mehr als $22 \%$ einen Testosteronwert unter $8 \mathrm{nmol} / \mathrm{l}$ hatten.

Dr. Udo Bermes, Facharzt für Urologie, Wiesbaden, betonte: „Viele Männer, mit denen wir gesprochen haben, sehen wir gar nicht oder nur selten zur Gesundheitsvorsorge in Klinik und Praxis. Deshalb finde ich es gut, dass so viele nun die Möglichkeit genutzt haben, sich bei der Aktion der TestovitalMess-Bar zu informieren und checken zu lassen."

Hypogonadismus ist häufig ein Indikator für zugrunde liegende Erkrankungen wie Herz-Kreislauf-Probleme, das metabolische Syndrom oder Typ-2-Diabetes. Eine aktuelle Studie der Gruppe von Wallaschofski fand sogar einen Zusammenhang zwischen erniedrigten Testosteronwerten und einem erhöhten Mortalitätsrisiko[Haring R et al. Eur Heart J 2010; 31: 1494-501]. Die Behandlung des Hypogonadismus kann mit Testosteronpräparaten erfolgen. Neben einem Testosteron-Gel (z.B. Testogel ${ }^{\oplus}$ ) das täglich vom Patienten selbst auf Oberarme, Schultern oder Bauch appliziert wird, steht die intramuskuläre Depotspritze Nebido ${ }^{\oplus}$ zur Verfügung, die den Testosteron-Spiegel über etwa 10-14 Wochen im Normbereich hält.

Nach Informationen von

Bayer HealthCare, Leverkusen 